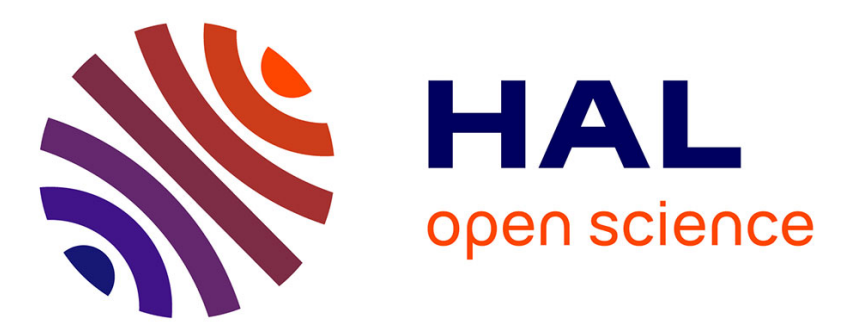

\title{
Réparer l'exploitation sexuelle. Le dispositif d'indemnisation des victimes de traite en France
}

Milena Jakšić, Nadège Ragaru

\section{To cite this version:}

Milena Jakšić, Nadège Ragaru. Réparer l'exploitation sexuelle. Le dispositif d'indemnisation des victimes de traite en France. Cultures \& conflits, 2021, 122, pp.123-140. 10.4000/conflits.22965 . hal-03426656

\section{HAL Id: hal-03426656 https://hal.science/hal-03426656}

Submitted on 13 Dec 2021

HAL is a multi-disciplinary open access archive for the deposit and dissemination of scientific research documents, whether they are published or not. The documents may come from teaching and research institutions in France or abroad, or from public or private research centers.
L'archive ouverte pluridisciplinaire HAL, est destinée au dépôt et à la diffusion de documents scientifiques de niveau recherche, publiés ou non, émanant des établissements d'enseignement et de recherche français ou étrangers, des laboratoires publics ou privés. 
(version auteures - pour le texte publié, se reporter à : https://www.cairn.info/revue-cultures-et-conflits-2021-2-page-123.htm

\title{
Réparer l'exploitation sexuelle \\ Le dispositif d'indemnisation des victimes de traite en France
}

Milena Jakšić et Nadege Ragaru

Cultures \& Conflits, 2021/2 (n 122), p.123-140

\begin{abstract}
Résumé : À partir d'une enquête consacrée au dispositif d'indemnisation des victimes de traite, cet article interroge le travail normatif et les contraintes professionnelles à l'œuvre dans la mise en barème des souffrances endurées par les victimes. En suivant les travaux de Viviana Zelizer sur les pratiques de marquage de l'argent, l'article montre que l'argent public alloué aux victimes de traite est loin d'être socialement neutre. Il fait au contraire l'objet d'un encadrement institutionnel spécifique et suscite de nombreuses disputes quant à sa juste attribution. L'article montre cependant que malgré les divergences, les acteurs impliqués dans le processus d'indemnisation parviennent à transformer la signification sociale de l'argent issu du proxénétisme et de la traite. L'argent "sale » de l'exploitation devient vertueux une fois alloué aux victimes de traite. Le processus de victimisation n'efface cependant pas le stigmate de prostituée et de sans-papiers associé aux victimes, justifiant leur contrôle et surveillance au-delà de l'épreuve d'indemnisation.
\end{abstract}

Mots clés : France, traite des êtres humains, réparation, sociologie, 21è siècle.

Repairing sexual exploitation. The compensation system for victims of human trafficking in France

Summary : Based on a study of administrative compensation accorded to victims of sex trafficking, this article examines the normative work and professional constraints that go into the evaluation of the suffering endured by prostitutes. Inspired by Viviana Zelizer's work on the earmarking practices affecting money, we show that sums allocated to victims of trafficking are given a specific value in the process. They go through institutional procedures, at each step of which their fair use, as public money, gives rise to disputes. The article also demonstrates how, through these conflicts, the actors involved in the compensation process collectively succeed in transforming the social significance of the money obtained from sex trafficking. The "dirty" money made by exploiting women becomes virtuous after having been allocated to the victims of trafficking. Acknowledging their victimhood, however, does not erase the stigma weighing on the women as both ex-prostitutes and undocumented migrants, justifying continued oversight even after the victims have endured the ordeal of going through the reparation process.

Key words : France, human trafficking, reparation, sociology, 21t century.

Milena Jakšić est sociologue, chargée de recherche au CNRS à l'Institut des sciences sociales du politique (ISP, Université Paris Ouest Nanterre La Défense, ENS Paris-Saclay). Après avoir travaillé sur les politiques de lutte contre la traite des êtres humains en France, ses recherches actuelles portent sur le traitement judiciaire de l'enfance en guerre par les tribunaux pénaux internationaux. Elle a notamment publié : La traite des êtres humains en 
France. De la victime idéale à la victime coupable, Paris, CNRS éditions, 2016 ; "Trouver l'enfant soldat. L'enquête judiciaire dans l'affaire Thomas Lubanga Dyilo », Terrain, 2019. Email :mjaksic@parisnanterre.fr

Nadège Ragaru est directrice de recherche et enseignante à Sciences Po Paris (CERI-CNRS). Ses recherches actuelles portent sur la sociologie des procès (procès pour crimes de guerre ; procès au titre de la traite des êtres humains), ainsi que sur l'histoire et l'historiographie du communisme et de la Shoah en Europe du sud-est. Ses publications récentes comprennent : (dossier dir.) " Écritures visuelles, sonores et textuelles de la justice : une autre histoire des procès à l'Est », Cahiers du monde russe (61/3-4, 2020), p. 275-498 ; « Et les Juifs bulgares furent sauvés... » Une histoire des savoirs sur la Shoah en Bulgarie, Paris : Presses de Sciences Po, 2020 ; (dossier dir. avec Milena Jakšić) « La victime au tribunal : du témoignage à la preuve judiciaire ", Droit et société, 102, 2ème trim., 2019, p. 227-318. Email : nadege.ragaru@sciencespo.fr

Les $^{1}$ fonds d'indemnisation des victimes des dommages corporels et psychiques n'ont cessé de se multiplier ces dernières décennies en France, comme si chaque nouvel accident, chaque nouvelle catastrophe, chaque nouvelle infraction, appelait une nouvelle réponse en termes de réparation des malheurs ${ }^{2}$. Le foisonnement de ces dispositifs n'est assurément pas propre à la France. Aux États-Unis, la prise en compte des victimes a entraîné le développement d'un droit de compensation des dommages (Tort law) : l'enjeu n'est plus seulement de qualifier la faute (naming) et de rechercher un coupable (blaming), encore fautil fixer le juste prix du préjudice subi (claiming) et réclamer à l'offenseur une compensation monétaire ${ }^{3}$. Aux États-Unis comme en France, il est devenu également possible d'obtenir un dédommagement financier sans imputation de faute (no fault). Cette évolution des représentations et des pratiques de prises en charge des victimes a pris la forme d'un nouveau séquençage de l'accès aux droits des personnes ordinaires, au sein duquel les dispositifs d'indemnisation constituent l'aboutissement de ce long processus de victimisation, mais présentent aussi de nombreux obstacles pour les personnes. Dans bien des situations, cependant, parmi les potentiels destinataires de ces nouvelles institutions, le non-recours au droit l'emporte sur le recours. Selon une logique tant de fois étayée empiriquement, plus les épreuves sont nombreuses et plus les personnes renoncent à réclamer leur dû. De fait, la grande majorité des dispositifs d'indemnisation des dernières années a été le fruit de mobilisations collectives ${ }^{4}$ : pour faire entendre leur voix, les victimes ont été amenées à se

\footnotetext{
${ }^{1}$ Nous remercions Janine Barbot, les membres de l'ANR ProsCrim ainsi que les évaluateurs anonymes de la revue pour leurs précieux commentaires sur la version antérieure du texte.

${ }^{2} \mathrm{Si}$ les dispositifs juridiques et institutionnels destinés à assurer une réparation monétaire des malheurs ont connu un développement très sensible ces dernières décennies, ils viennent s'inscrire dans une historicité plus longue, qui correspond à la naissance, dans les pays occidentaux, des sociétés d'assurances et à l'émergence de pratiques d'estimation d'un «prix de la vie ». Ces transformations sont étudiées par François Ewald à partir de la loi de 1898 sur l'indemnisation des accidents du travail. L'auteur montre que l'indemnisation des dommages corporels résulte d'une transformation profonde du droit des responsabilités et de l'émergence des assurances privées. L'indemnisation « sans faute » est créée dans le but d'empêcher les ouvriers d'intenter des procès à leurs patrons. Cf. Ewald F., L'État providence, Paris, Grasset, 1986.

3 Felstiner W.F., Abel R., Sarat A., «The emergence and transformation of disputes : naming, blaming, claming », Law and Society Review, 15, 3/4, 1980-1981, pp. 631-654 (Traduction française, 1991, «L’émergence et la transformation des litiges : réaliser, reprocher, réclamer », Politix, n¹6, pp. 41-54).

${ }^{4}$ Pour ne citer qu'un exemple : le Fonds de garantie des victimes d'actes de terrorisme est créé sous l'impulsion de Françoise Rudetzky et de son association SOS attentats. Cf. Fassin D., Rechtman R., L'empire du traumatisme. Enquête sur la condition de victime, Paris, Flammarion, 2007.
} 
constituer en collectifs hybrides composés de « victimisateurs ${ }^{5} »-$ avocats, experts, médecins, familles, journalistes - susceptibles d'assurer le succès de leur cause.

Cette multiplication des causes victimaires a donné lieu à une littérature pléthorique en sciences sociales. Les terrains d'investigation empiriques sont presque aussi nombreux que les collectifs de victimes mobilisés. Que ce soit les accidents de la route ${ }^{6}$, les erreurs médicales ${ }^{7}$, les catastrophes industrielles et naturelles ${ }^{8}$, les maladies professionnelles ${ }^{9}$ ou encore les attentats, les chercheurs en sciences sociales ont trouvé ici un terrain d'étude propice pour explorer l'accès des personnes ordinaires à la justice. Les collectifs des victimes ont été abordés sous l'angle de la sociologie des mobilisations ${ }^{10}$, de l'inégalité sociale dans l'accès aux droits ${ }^{11}$, des opérations de constitution de la plainte ${ }^{12}$ ou encore des « attentes normatives structurant le jugement ${ }^{13} »$. Indépendamment des approches privilégiées, toutes ces recherches concourent au constat de nombreux obstacles matériels et cognitifs devant être franchis pour officialiser le statut de victime. Plus spécifiquement, les recherches portant sur les essais nucléaires français, les agriculteurs empoisonnés par les pesticides ou les cancers du travail, reviennent dans le détail sur les difficultés pour les victimes à établir un lien de causalité entre la dégradation de leur état de santé et l'exposition au risque industriel. Pour obtenir gain de cause, les victimes doivent se livrer à un véritable « travail étiologique ${ }^{14}$ » en recueillant les données médicales et statistiques susceptibles de prouver, de manière certaine et convaincante, le lien de causalité entre l'exposition au risque et la survenue de problèmes de santé. Dans le cas des cancers du travail touchant principalement des personnes issues des classes populaires, c'est le non-recours au droit qui prévaut. Le refus de demande d'indemnisation est souvent justifié par la crainte d'être assimilés à des «mendiants » réclamant « l'aumône $»{ }^{15}$.

\footnotetext{
${ }^{5}$ Barthe Y., Les retombées du passé. Le paradoxe de la victime, Paris, Seuil, 2017.

${ }^{6}$ Ponet Ph., « Remettre les corps en ordre : entre savoirs et pouvoirs. La «professionnalisation » de l'évaluation médicale du dommage corporel », Revue française de sociologie, vol. 48, n 3, 2007, pp. 477-517.

${ }^{7}$ Barbot J., Dodier N., « De la douleur au droit : ethnographie des plaidoiries lors de l'audience pénale du procès de l'hormone de croissance contaminée », in Berger M., Cefaï D., Gayet-Viaud C. (dir.), Du civil au politique. Ethnographies du vivre ensemble, Bruxelles, Peter Lang, 2011, pp. 289-322 ; Fillion E., À l'épreuve du sang contaminé. Pour une sociologie des affaires médicales, Paris, EHESS, 2009. Fillion E., Torny D., «De la réparation individuelle à l'élaboration d'une cause collective. L'engagement judiciaire des victimes du distilbène », Revue française de science politique, vol. 65, n 4, 2015, pp. 583-607.

${ }^{8}$ Centemeri L., « Retour à Seveso. La complexité morale et politique du dommage à l'environnement », Annales. Histoire, Sciences Sociales, vol. 66e année, n 1, 2011, pp. 213-240; Henry E., «Intéresser les tribunaux à sa cause : contournement de la difficile judiciarisation du problème de l'amiante ", Sociétés contemporaines, $\mathrm{n}^{\circ} 52$, 2003, pp. 39-59 ; Jobin P., Maladies industrielles et renouveau syndical au Japon, Paris, Ehess, 2006 ; Jouzel J.N., Prete G., « Devenir victime des pesticides : le recours au droit et ses effets sur la mobilisation des agriculteurs phyto-victimes », Sociologie du travail, ${ }^{\circ} 56$ (4), 2014, pp. 435-453.

${ }^{9}$ Marichalar P., Qui a tué les verriers de Givors? Une enquête de sciences sociales, La Découverte, Paris, 2017.

${ }^{10}$ Lefranc S., Mathieu L., Mobilisations de victimes, Rennes, Presses Universitaires de Rennes, coll. « Res publica », 2009 ; Vilain J.-P., Lemieux C., « La mobilisation des victimes d'accidents collectifs : vers la notion de "groupe circonstanciel" », Politix, 44, 1998, pp. 135-160

${ }^{11}$ Galanter M., " Pourquoi les mêmes gardent l'avantage? Introduction à la traduction française », Droit et société, vol. 85, n³, 2013, pp. 559-574.

${ }^{12}$ Barbot, J., Winance, M. \& Parizot, I., « Imputer, reprocher, demander réparation. Une sociologie de la plainte en matière médicale », Sciences sociales et santé, n² 2(2), 2015, pp. 77-105.

${ }^{13}$ Barbot J., Dodier N., « Face à l'extension des indemnisations non judiciaires. Le cas des victimes d'un drame de santé publique », Droit et société, vol. 89, n 1, 2015, pp. 89-103.

${ }^{14}$ Jouzel J.-N., Prete G., « De l'intoxication à l'indignation. Le long parcours d'une victime des pesticides », terrains \& travaux, vol. 22, $\mathrm{n}^{\circ} 1,2013$, pp. 59-76.

${ }_{15}$ Marchand A., «Quand les cancers du travail échappent à la reconnaissance. Les facteurs du non-recours au droit », Sociétés contemporaines, vol. 102, n² 2016, pp. 103-128.
} 
$\mathrm{Au}$ regard des configurations étudiées dans cette littérature abondante, les victimes de traite présentent certaines caractéristiques qui font de ce groupe un cas à part, et confèrent aux dispositifs d'indemnisation dont elles bénéficient leur singularité. Contrairement aux victimes des catastrophes industrielles ou naturelles, les préjudices qu'elles disent avoir subis ne résultent pas d'un événement isolé. C'est une condition sociale - la prostitution - dénoncée comme dégradante pour les femmes ${ }^{16}$, qu'il est question ici de réparer au nom de la solidarité nationale. Cela nous conduit à une seconde particularité des victimes de traite : le processus de victimisation n'efface pas le stigmate de prostituée. L'activité prostitutionnelle a été associée, depuis le XIXe siècle ${ }^{17}$, à une notion de souillure. Cette représentation, à l'origine de nombreux désaccords au sein même des instances en charge de la réparation, complique le travail d'indemnisation : elle nourrit en effet les controverses relatives à un « juste » usage de l'argent public destiné à des personnes prostituées, aux étrangères en situation irrégulière sans perspective d'un avenir professionnel stable. Doutes, hésitations, voire contestations, sont perceptibles à toutes les étapes du processus de réparation. Aux yeux de certains acteurs impliqués dans les demandes d'indemnisation, les victimes de traite présentent ces « inutiles au monde ${ }^{18} »$ qu'il faut à la fois secourir et surveiller, l'enjeu étant aussi bien de contrôler les sommes allouées que les manières de les dépenser. Cette tendance va dans le sens des travaux de Viviana Zelizer pour qui l'argent est loin d'être « culturellement neutre » et « socialement anonyme ${ }^{19}$. Celui des pauvres est toujours l'objet d'un « marquage », d'un jugement, d'une instruction. En cela aussi, et c'est le troisième point, les victimes de traite se distinguent d'autres catégories de victimes. Dans les affaires d'erreurs médicales ou de catastrophes naturelles, l'usage privé de l'argent public ne suscite aucune surveillance particulière. Celui alloué aux victimes de traite fait au contraire l'objet d'un encadrement, parfois au-delà de l'épreuve d'indemnisation, comme si l'argent représentait une «forme dangereuse de secours $»^{20}$.

Dans ce contexte, cet article se propose d'explorer plus spécifiquement une des facettes du processus d'indemnisation des victimes de traite, à savoir le travail normatif et les contraintes professionnelles à l'œuvre dans la «mise en barème ${ }^{21}$ » des souffrances endurées par les victimes de traite. L'objectif est de regarder comment le traitement d'une activité jugée socialement dégradante façonne les débats associés à l'usage de l'argent public et les pratiques de dédommagement. L'enquête met en présence trois catégories d'acteurs impliqués dans ce processus. D'un côté, les avocats des victimes, chargés de formuler une demande d'indemnisation en chiffrant chaque poste de préjudice. De l'autre, le Fonds de garantie, un organisme opérant pour 1'Etat avec pour mission de veiller à la juste distribution des ressources collectives. Entre les deux, se situent les Commissions d'indemnisation des victimes d'infraction (CIVI), ces tribunaux civils créés en 1983 par une réforme du droit pénal en vue d'indemniser des préjudices jusqu'alors non couverts, soit parce que l'auteur des faits était inconnu ou insolvable, soit parce que les faits ne pouvaient pas être couverts par d'autres organismes (sécurité sociale, assurances, mutuelles). Placés en position d'arbitre entre le Fonds de garantie - responsable de la juste distribution de l'argent public - et les avocats des victimes - défenseurs d'intérêts privés - les CIVI sont appelées à trancher des litiges et à fixer le montant d'indemnisation pour chaque poste de préjudice. À chaque étape du processus, les

\footnotetext{
${ }^{16}$ La dénonciation est portée par les mouvements abolitionnistes et une partie du « féminisme d'État». Cf. AUTEURE.

${ }^{17}$ Corbin A., Les filles de noce. Misère sexuelle et prostitution, XIXe et XXe siècles, Paris, Flammarion, 2015 [1 $1^{\text {èr }}$ édition 1979].

${ }^{18}$ Castel R., Les métamorphoses de la question sociale. Une chronique du salariat, Paris, Fayard, 1995.

${ }^{19}$ Zelizer V., La signification sociale de l'argent, Paris, Seuil, 2005, p. 51.

${ }^{20}$ Ibid., p. 202.

${ }^{21}$ Sayn I., (dir.), Le droit mis en barèmes ?, Paris, Dalloz, 2014.
} 
experts psychologues et gynécologues sont convoqués par les deux parties. Leur rôle est de déterminer, sur la base d'un examen médical, le lien de causalité entre activité prostitutionnelle et pathologies constatées. C'est sur la base de leurs rapports que les avocats des victimes proposent une compensation monétaire. Celle-ci est systématiquement revue à la baisse par les experts du Fonds de garantie, tenants d'une approche non-dépensière de l'argent public. Les désaccords entre les deux parties, qui se jouent à coup d'appels et de contreappels, contribuent à l'étirement dans le temps de la durée moyenne du processus d'indemnisation, laquelle est d'environ cinq ans.

L'analyse s'appuie sur trois types de sources recueillies au cours d'une enquête de plus de quinze ans : des expertises médicales sollicitées à différentes étapes de la demande de réparation; des décisions des Commissions d'indemnisation des victimes d'infractions; des conclusions des avocats des victimes et du Fonds de garantie. Ce matériau est complété par des entretiens réalisés entre 2014 et 2016 avec des avocats des victimes, des experts judiciaires et des travailleurs sociaux opérant au sein d'associations spécialisées dans la prise en charge des personnes prostituées.

Centré sur les jugements moraux et les logiques professionnelles au fondement de ces désaccords, l'article montre comment, malgré leurs divergences, les acteurs impliqués dans le processus d'indemnisation parviennent à transformer la signification sociale de l'argent issu du proxénétisme et de la traite : l'argent « sale » de l'exploitation devient " vertueux » par une série de cadrages institutionnels destinés à redresser des vies abîmées.

\section{Aux sources du dispositif de réparation : un tout petit monde}

Dans un article consacré aux réactions des individus face au drame sanitaire qui les touche, Janine Barbot et Nicolas Dodier identifient quatre types de dispositifs susceptibles d'être mobilisés à des fins de réparation des malheurs : judicaire, d'indemnisation extrajudiciaire, médiatique et associatif. Se focalisant pour leur part sur le seul dispositif judiciaire, ils veulent étudier "l'ensemble des interactions entre les victimes et ces dispositifs de réparation » en regardant non-seulement comment les dispositifs façonnent les demandes des individus, mais également, comment, en retour, les individus transforment les dispositifs en question en les critiquant, en les contournant ou en les mettant à distance ${ }^{22}$. Cette proposition, aussi stimulante soit-elle, s'applique difficilement au cas qui nous occupe ici. Nous sommes en effet très loin d'une opposition binaire entre collectifs de victimes et dispositifs d'indemnisation. Le destin d'une demande de réparation au titre de traite dépend d'un nombre limité de personnes. Loin d'être portées par des collectifs de victimes ou des associations, les demandes relèvent principalement des relations de proximité (sociale, professionnelle, générationnelle) entre quelques individus pour qui la traite représente à la fois un investissement professionnel et un engagement social et militant. En reconstituant la trajectoire de leur engagement dans un dispositif peu mobilisé, nous voulons mettre l'accent sur la capacité des acteurs à transformer la signification sociale de l'argent. Ici, l'argent « sale » du proxénétisme et de traite se transforme, grâce au travail critique des acteurs, en argent « vertueux » destiné à réparer les dommages causés par la traite.

\section{Un dispositif peu mobilisé}

L'ajout de la traite sur la liste des infractions couvertes par les CIVI est relativement récent. Elle y est intégrée en mars 2004, un an après son entrée dans le code pénal français

\footnotetext{
${ }^{22}$ Barbot J., Dodier N., « Se confronter à l'action judiciaire », L'Homme [En ligne], n 223-224, 2017, p. 101.
} 
(loi du 18 mars 2003), mais il faut attendre 2008 pour qu'un premier dossier d'indemnisation soit instruit devant un tribunal de province ${ }^{23}$. Cette tendance se poursuit dans la décennie suivante, avec seulement cinq décisions rendues par des cours d'appel de Rennes, de Nantes et de Marseille ${ }^{24}$. On doit cette faible sollicitation des CIVI au nombre infime de condamnations pour traite des êtres humains en France ${ }^{25}$, les magistrats préférant retenir la qualification du "proxénétisme aggravé » qui, elle, ne figure pas sur la liste des infractions visées par les CIVI. Les associations, quant à elles, sont peu familières des démarches de réparation. Davantage préoccupées par l'accompagnement social et administratif des victimes (obtention d'un titre de séjour, recherche d'un travail, prise en charge médicale), elles s'engagent peu dans la voie pénale (dépôt de plainte, identification des coupables) et formulent encore moins des demandes de réparation.

Ce faible recours aux CIVI peut paraître étonnant compte tenu des conditions relativement souples pour prétendre à une indemnisation. Il suffit en effet de justifier d'une incapacité totale de travail (ITT) ou d'une incapacité permanente partielle (IPP) supérieure à un mois. La charge de la preuve est considérablement allégée, les requérants n'ayant pas à prouver l'insovabilité des auteurs, ni à démontrer les atteintes portées à leurs conditions de vie en cas d'infraction graves. Les sommes pouvant être demandées « sont déplafonnées » ${ }^{26}$ et les délais pour saisir les CIVI restent relativement larges : trois ans suivant l'infraction ou la décision pénale, alors que celui-ci était fixé à un an dans les années 1980. Enfin, et c'est crucial pour les victimes de traite qui peinent à obtenir un titre de séjour : la loi du 5 août 2013 autorise les étrangers en situation irrégulière à saisir les CIVI, un droit qui se limitait jusqu'alors aux seules personnes de nationalité française ou aux étrangers en situation régulière.

Une avocate et une travailleuse sociale engagées

Cet effort législatif pour intégrer la traite à la liste des infractions visées par les CIVI n'aboutit donc pas à un afflux des demandes. En 2008, l'examen du premier dossier devant un tribunal de province ne suscite aucune attention particulière, alors même que la requérante, par l'intermédiaire de son avocate, obtient gain de cause et que l'issue de la demande marque un tournant dans le traitement des dossiers de traite. La réussite de l'affaire n'est attribuable ni à la mobilisation du milieu associatif, ni à la médiatisation de la souffrance. Elle n'est pas non plus «vecteur de publicisation ${ }^{27}$ » du problème social incarné par la traite. Discrétion et tâtonnement, mais aussi innovation dans le domaine judiciaire, caractérisent cette première demande.

En l'absence des collectifs de victimes et de médiatisation, le dossier est instruit par une avocate, Claire B., jusqu'alors spécialisée dans le droit de la famille et des violences faites aux femmes. Qualifiée de "pasionaria du barreau » par un collègue juge dont il sera question plus loin, elle voit dans les dossiers de traite moins un travail à accomplir qu'une

\footnotetext{
${ }^{23}$ Et ce, non avec le qualificatif de « traite », mais avec celui de « proxénétisme aggravé », nous y reviendrons.

${ }^{24}$ MIPROF, Rapport à la Commission européenne sur la lutte contre la traite des êtres humains, 2011-2014, 2014.

${ }^{25}$ AUTEURE XXXXX.

${ }^{26}$ GISTI, Indemnisation des victimes d'infractions, décembre 2011, p. 4.

${ }^{27}$ Sur le droit comme « vecteur de publicisation » cf. Henry E., « Le droit comme vecteur de publicisation des problèmes sociaux: effets publics du recours au droit dans le cas de l'amiante », in Israël L., Sacriste G., Vauchez A., Willemez L. (dir.), Sur la portée sociale du droit. Usages et légitimité du registre juridique, Paris, PUF, 2005, pp. 187-200.
} 
cause à défendre. À l'instar des « cause lawyers $^{28}$ », le droit devient à la fois l'instrument et l'objet de la cause. Dans le récit de soi qu'elle offre lors d'un entretien réalisé en décembre 2014, cette rhétorique de l'engagement se prolonge dans le mode d'organisation de son cabinet, situé dans une ville de province et composé essentiellement d'avocates femmes. Le droit de la famille reste en effet « l'un des domaines où la féminisation du barreau est la plus visible $\gg{ }^{29}$. Dans l'activité du cabinet de Claire B., les dossiers relatifs au droit de la famille et aux violences faites aux femmes l'emportent pourtant sur les affaires de traite qui ne représentent que $10 \%$ de l'ensemble du contentieux. À l'inverse, ces dernières sont plus rémunératrices en termes de chiffre d'affaires.

Il n'est pas sans intérêt de revenir ici sur la restitution, par cette avocate, de sa trajectoire d'engagement dans la mesure où celle-ci participe de la définition qu'elle donne à voir une trajectoire sociale minoritaire au sein d'un barreau où prévalent les univers sociaux marqués par la détention d'un capital économique et/ou d'une appartenance aux professions libérales. Son investissement dans la cause des femmes, Claire B. l'inscrit dans une histoire familiale «engagée » et «féministe »: «Moi, je viens d'un milieu militant, de gauche, syndiqué, des parents profs, une mère féministe. Un père féministe aussi d'ailleurs à sa manière. Mais voilà, ça s'est fait par hasard, les choses qui m'ont conduite là, et avec une prise de conscience vraiment d'inégalités hommes/femmes devant la justice. Et puis, ça m'éclate, ça m'intéresse, je trouve ça passionnant, ça donne du sens $»^{30}$. Les débuts de son parcours professionnel sont pourtant très éloignés des thématiques liées à la cause des femmes. Après des études en commerce international, elle obtient un DESS en droit humanitaire et travaille pour l'OSCE pendant deux ans à Sarajevo. De retour en France, en 2000 elle s'inscrit en études de droit et passe le concours d'entrée au barreau un an plus tard. Qu'elle choisisse de se spécialiser, en tant qu'avocate, dans le droit de la famille et la violence faite aux femmes est référé, dans la reconstitution qu'elle offre de son parcours, à cet engagement féministe. Elle investit le terrain de la traite à partir de 2005, alors que les transformations législatives sont à leur apogée.

Son intérêt grandissant pour le sujet est concomitant de l'arrivée d'Anna D. au sein d'une association de santé communautaire en charge de l'accompagnement des personnes prostituées et des victimes de traite. Issue de la classe moyenne, Anna D. grandit en banlieue parisienne et rejoint l'association de santé communautaire en première année de thèse de géographie qu'elle entame à l'Université de la Sorbonne, et qu'elle décide d'abandonner, sans regrets, pour s'engager à temps plein dans le travail associatif. Au fil des années, Anna D. est parvenue à acquérir un véritable savoir-faire en matière du suivi des victimes de traite d'abord à Paris, puis en France. On peut même parler de monopole, tant en termes d'accompagnement social et administratif des victimes que du nombre des affaires dans lesquelles les victimes se constituent partie-civiles. Pendant près de dix ans, la quasi-totalité des victimes qu'elle suit est orientée vers Claire B., entre autres dans les dossiers d'indemnisation. Cette dernière parvient à assoir son autorité dans le champ en raison du nombre de litiges tranchés en sa faveur : elle est désormais sollicitée par l'ensemble des associations investies sur le terrain de la traite, indépendamment de leur position idéologique sur le sujet (Médecins du Monde, Ippo, le dispositif Ac.Sé). L'avocate elle-même prend soin de rester en dehors de ces clivages normatifs : elle ne se déclare ni abolitionniste ni militante pro travail du sexe.

\footnotetext{
${ }^{28}$ Sarat A., Scheingold A. S., Cause Lawyering. Political Commitments and Professional Responsibilities, Oxford University Press, New York, 1998.

${ }^{29}$ Bessière C., Mille M., Schütz G., «Les avocat·es en droit de la famille face à leur clientèle. Variations sociales dans la normalisation de la vie privée », Sociologie du travail, Vol. 62, n 3, 2020, p. 5.

${ }^{30}$ Entretien réalisé le 12 décembre 2014.
} 
Le recours au dispositif d'indemnisation est donc moins le résultat d'un engagement collectif que de relations tissées entre une avocate pénaliste spécialisée dans le droit de l'adoption et de la famille, et une travailleuse sociale qui détient une large partie des dossiers de traite. Les intérêts communs, plus que l'amitié, ont façonné leurs relations de travail, tandis que la proximité sociale, générationnelle et politique a facilité leur collaboration.

Le juge, un allié

La connivence professionnelle entre les deux femmes n'explique pas à elle seule la réussite de leur démarche. Le cabinet de Claire B. est situé dans une ville de province de taille moyenne où les relations entre juges et avocats, faites de familiarité mais aussi de tensions, ne sont pas sans effet sur l'issue des affaires ${ }^{31}$. Chacun y est précédé de sa réputation. Dans cette ville de province, la CIVI est d'une toute petite taille, et au moment de notre enquête, elle est présidée par un juge de 65 ans proche de la retraite. Un rendez-vous avec celui-ci est obtenu par l'intermédiaire de cette dernière, qui nous met cependant en garde, tout comme d'autres avocates de son cabinet. Claude J. serait connu pour donner « trop facilement les compliments aux jeunes femmes » et peut être «lourd des fois ». Forte de cette mise en garde, nous avons abordé l'entretien avec une certaine prudence. Claude J. s'est pourtant révélé davantage aidant et cordial qu'aguicheur. Pendant toute la durée de l'entretien, il est sorti à plusieurs reprises de son cabinet pour faire des photocopies des décisions rendues par la CIVI. Trois éléments ressortent de l'entretien. Une certaine fascination qu'il semble éprouver pour Claire B., qu'il décrit, nous l'avons dit, de "pasionaria du barreau ». Claire B. est d'avis de tous " une très belle femme », qui n'hésite pas à défier les juges pour obtenir gain de cause. Claude J. nourrit à son égard un sentiment protecteur et donne l'impression, tout au long de l'entretien, d'avoir toujours pris sa défense dans les désaccords qui l'ont opposée au Fonds de garantie. Le juge va jusqu'à dire : " J'avais peur pour la santé de Claire B. parce qu'elle peut être éruptive. Je l'appelle 'pasionaria du barreau', elle sait que je l'appelle comme ça ${ }^{32} »$. Plus loin dans l'entretien, il revient longuement sur l'attitude jugée agressive du Fonds à l'égard de Claire B. : « Le Fonds de garantie voit rouge quand ils voient Claire B. Il est très offensif avec elle, pour ne pas dire c'est vraiment limite de la grossièreté parfois ». Les avocats du Fonds contestent en effet systématiquement les sommes réclamées par l'avocate qu'ils considèrent comme « excessives ».

Cette attitude protectrice du juge contraste avec le sentiment de défiance qu'il nourrit à l'égard du Fonds de garantie. Il qualifie leur position de « restrictive » et ajoute : « Si je veux être grossier, je dirais que le Fonds de garantie a une position qui consiste à faire le moins de dépenses budgétaires possibles. [...] parfois il a même eu des positions outrancières ». Il va jusqu'à dénoncer les "préjugés » du Fonds qui ne souhaite pas, d'après lui, dépenser l'argent public pour rembourser les produits issus de la prostitution. Cette position consistant à veiller au bon usage de l'argent public serait partagée par certains de ses collègues juges qui se demandent pourquoi une victime de traite percevrait dix fois plus de dédommagement qu'une victime de viol. Là encore, Claude J. dit sa désapprobation et nous expose sa vision de la prostitution, qu'il assimile aux viols à répétition : "Une femme a été violée, c'est un crime grave, c'est une atteinte à l'intégrité physique etc. Mais pour les prostituées qui ont passé deux, trois ans enfermées entre quatre murs, entraînées à avoir du rendement à la demande, en ayant aboli toute vie personnelle, en étant avortées de force lorsqu'elles attendaient un enfant. C'est une déstructuration complète ». Conscient que tous ses collègues ne partagent pas sa

\footnotetext{
${ }^{31}$ Pour un autre exemple des effets de la proximité sur l'issue des affaires, voir Le Collectif Onze, Au tribunal des couples. Enquête sur des affaires familiales, Paris, Odile Jacob, 2013.

${ }^{32}$ Entretien réalisé le 12 décembre 2014.
} 
vision de la prostitution comme dégradation ultime du corps des femmes, il craint la sévérité de son successeur dans l'examen des demandes d'indemnisation.

\section{Transformer la signification de l'argent}

Connivence professionnelle et entente sur ce qui doit être réparé aboutissent à l'instruction d'une première demande d'indemnisation pour traite en 2008. La demande est formulée par Claire B. devant la CIVI présidée par Claude J. La victime, elle, est accompagnée par Anna D. qui l'encourage à faire une demande d'indemnisation. Or, la qualification juridique retenue par le parquet dans ce dossier est celle du "proxénétisme aggravé », non de traite, ce qui l'exclut a priori du droit à la réparation, le proxénétisme aggravé ne faisant pas partie des infractions visées par les CIVI. Pour contourner cet obstacle, Claire B. est obligée d'innover. Le monde des dispositifs d'indemnisation est en effet peuplé de ces "découvreurs » et « innovateurs », comme l'ont très bien montré Stéphane Latté et Richard Rechtman dans leur enquête sur l'explosion de l'usine AZF à Toulouse ${ }^{33}$. Dans le cas de la traite, on observe la même nécessité d'innovation pour dédommager ce qui était jusqu'alors exclu du droit à la réparation.

Ici, l'avocate procède en plusieurs étapes. D'abord, elle déplace le registre argumentatif depuis la question de la nature de l'infraction vers celle de ses conséquences physiques. Ce n'est plus la désignation de l'infraction qui compte ( " proxénétisme aggravé » ou « traite »), mais les traces physiques et psychologiques laissées sur la victime. Forte du soutien du président de la CIVI, l'avocate se montre audacieuse, expérimente et plaide pour une incapacité temporaire du travail (ITT) supérieure à un mois alors qu'elle ne dispose d'aucune preuve matérielle allant en ce sens : « Je n'avais pas de certificats médicaux qui disaient que j'avais une ITT de trente jours ou une incapacité physique permanente. Donc, j'y suis allée un peu au bluff en disant devant la CIVI, voilà, il y a cette situation, certes, le proxénétisme ne fait pas partie des infractions qui peuvent être réparées devant la CIVI mais, moi, je considère qu'il faut que vous mandatiez les expert.e.s qui puissent nous dire si cette jeune fille elle a une IPP ou une ITT supérieure à trente jours. Et puis [le magistrat] (...) m'a suivie. Et il a désigné les expert.e.s. Et puis après, j'ai travaillé avec des expert.e.s qui ont été amenés à considérer que la période d'exploitation sexuelle était de l'incapacité temporaire du travail. Et donc, là, c'était tout à fait possible, j'avais un boulevard qui s'ouvrait devant moi pour aller devant la CIVI et faire liquider mes préjudices ${ }^{34}$. » Il s'agit moins ici de « bluff» que de mettre à profit les liens de connivence noués avec le juge président. Claire B. sait qu'elle a un allié de taille à la tête de la CIVI et la certitude de ce soutien l'encourage à prendre des risques.

Le coup de force qu'elle opère représente un véritable changement de paradigme puisqu'il requalifie toute la période d'activité prostitutionnelle en incapacité temporaire du travail et réclame des dédommagements en conséquence. S'alignant sur l'abolitionnisme dominant en France, l'avocate plaide en effet l'absence totale de libre arbitre pendant la période de contrainte prostitutionnelle : «Elle ne disposait pas de son corps, elle ne disposait pas de sa liberté d'aller et venir, elle ne disposait pas des bénéfices qu'elle pouvait tirer de la prostitution, et donc que c'était une période d'aliénation absolue qui devait se rapporter à l'ITT. Donc, ils ont accepté cette idée-là. »

\footnotetext{
${ }^{33}$ Le caractère inédit de la catastrophe a obligé les avocats à créer un nouveau « chef de réparation exceptionnel 'le préjudice spécifique' » visant moins à compenser « les conséquences socio-économiques de l'explosion » que le "'vécu psychologique' de ces dernières ». Cf. Latté S., Rechtman R., "Enquête sur les usages sociaux du traumatisme à la suite de l'accident 1 de l'usine AZF à Toulouse », Politix, vol. 73, no. 1, 2006, p. 179.

${ }^{34}$ Entretien Claire B., ibid.
} 
Claire B. ne s'arrête pas à cette première avancée et décide de créer un nouveau poste de préjudice qui rendrait compte du caractère spécifique du phénomène de traite : "le préjudice exceptionnel d'avilissement»: «L'idée, ça a été de considérer que cette exploitation sexuelle, alors quelle que soit la qualification pénale, traite des êtres humains ou proxénétisme, donc que cette exploitation sexuelle était à ce point avilissante, à ce point dégradante, à ce point attentatoire à la dignité qu'elle portait en elle l'idée d'une réparation particulière. » L'enjeu ici est de réparer l'atteinte à la dignité humaine causée par l'activité prostitutionnelle. L'avocate fait indirectement référence aux engagements internationaux de la France en matière de lutte contre la traite des êtres humains ${ }^{35}$. En créant ce nouveau poste de préjudice, elle traduit l'engagement législatif basé sur la compassion en dispositif de réparation.

Mais créer un préjudice spécifique à la traite ne suffit pas : il faut également trouver une méthode de calcul permettant d'en apprécier la valeur monétaire. Claire B. recourt alors à une décision de la Cour de cassation du 7 juin 1945 postulant qu'une prostituée est en droit de réclamer la restitution des sommes extorquées par son proxénète devant la jurisprudence civile. Le magistrat approuve la demande de Claire B., et sa cliente se voit restituer 80000 euros, soit l'équivalent des sommes versées à la proxénète. Le Fonds de garantie fait appel de la décision, mais la Cour d'appel de Paris, dans une décision rendue le 17 octobre 2013, donne finalement raison à l'avocate après avoir accepté la requalification du préjudice en « préjudice d'avilissement ». En créant ce nouveau poste de préjudice et en lui associant un mode de calcul spécifique, l'avocate ne se contente pas d'innover dans le domaine judiciaire. Elle transforme la signification sociale de l'argent issu du proxénétisme et de la traite. L'argent « impur » devient " pur» dès lors qu'il est destiné à faire le bien, à construire de nouveaux liens sociaux affranchis de toute contrainte et exploitation.

Cette transformation sociale de l'argent n'est pas sans susciter la fierté, tant de l'avocate que du juge. Ce dernier qualifie sa démarche «d'originale » et se félicite de la voir se généraliser à toutes les CIVI de France, y compris celles de Paris. Il se voit comme coproducteur, avec Claire B., d'un préjudice jusqu'alors inédit. Le Fonds de garantie, responsable de l'argent public, ne cesse pourtant de contester ce chef de préjudice selon les arguments qu'il convient maintenant de détailler.

\section{Les pratiques de marquage de l'argent public}

L'argent distribué ici n'est pas socialement neutre, il fait l'objet d'un processus de « marquage », pour reprendre le concept forgé par Viviana Zelizer. Ce concept désigne les différents usages de l'argent par les personnes engagées dans une transaction. En fonction des situations, l'argent peut servir de salaire, de don, de pourboire, d'aumône ou de prime. L'argent destiné aux victimes de traite ne relève ni de don, ni de charité, mais de dédommagement de ce qui fut brisé et qui peine à être reconstruit. Il est utilisé dans un but spécifique et fait l'objet d'un encadrement institutionnel particulier. Il engage la victime vis-àvis de l'institution distributrice qui a vocation de contrôler l'usage privé de l'argent public. Ce contrôle est d'autant plus grand qu'il s'agit des femmes pauvres suspectées d'incompétences en matière de dépenses.

\footnotetext{
${ }^{35}$ Suite à la ratification de la Convention des Nations unies contre la criminalité transnationale organisée, dite Convention de Palerme et du Protocole visant à prévenir, réprimer et punir la traite des personnes, en particulier des femmes et des enfants, additionnel (adoptée le 12 décembre 2000, ratifiée le 29 octobre 2002).
} 
Ces techniques de marquage ont leurs propres outils et instruments. Les experts s'appuient sur la nomenclature " Dintilhac », un instrument non contraignant proposant, depuis 2005, la réparation de 27 postes distincts divisés en deux grandes catégories : préjudices patrimoniaux (économiques) et extra-patrimoniaux (non-économiques). L'objectif de ce type de compartimentage est de couvrir tous les aspects de la vie, notamment ceux liés au travail et à la sexualité dans le cas de la traite. Ces notions n'étant pas définies par le droit, elles laissent une certaine latitude d'interprétation aux acteurs qui font intervenir leurs propres catégories morales en référence au travail et à la sexualité. Le processus de marquage est ainsi pris dans un double mouvement d'objectivation des préjudices à l'aide d'instruments de calcul standardisé et d'intrusion des catégories morales de jugement. Ainsi, le stigmate de prostituée et de sans-papiers ne disparait jamais complètement du processus de victimisation. On retrouve ses traces dans certains rapports d'experts. Cependant et contrairement aux travaux qui dénoncent la justice comme le lieu du «maintien de l'ordre social » et de re-production des hiérarchies entre les classes et les sexes ${ }^{36}$, nous préférons ici suspendre le jugement, pour insister davantage sur les compétences critiques des acteurs qui sont loin d'appliquer mécaniquement les normes juridiques. Pour le dire simplement: il n'y a pas d'un côté le Fonds de garantie doté d'un pouvoir irrévocable et de l'autre côté les victimes démunies face au droit. Une asymétrie existe de fait, comme dans toute relation engageant un donneur et un receveur, mais le receveur n'est pas pour autant dans une situation de contrainte absolue. Il peut toujours sortir de l'équation s'il l'estime nécessaire. L'exemple qui suit s'appuie sur un litige ordinaire d'un dossier de traite et vise à décrire simultanément les pratiques de marquage et de contre-marquage de l'argent de dédommagement. Si toutes les victimes finissent par obtenir réparations, celles-ci sont dans certains cas retardées du fait précisément de ces actes de contestation. L'ordre «dominant» n'est jamais fixé d'avance, il est constamment remis en question par les acteurs eux-mêmes.

\section{L'affaire : de la plainte à l'indemnisation}

Joy V. et Fatima M. sont deux femmes d'origine nigériane, arrivées en France en 2005 « pour y avoir une vie meilleure qu'en Afrique » et fuir la pauvreté. Aidées par les amis de la famille ${ }^{37}$, elles se plient, avant leur départ, à un rituel juju censé les protéger pendant leur traversée. Elles promettent en échange ne jamais dévoiler le nom des personnes ayant organisé leur voyage. Leurs ongles des pieds, une mèche de cheveux et des poils pubiens sont coupés et leurs vêtements confisqués par le sorcier qui les menaça de les tuer si «elles rompaient le pacte ». Trois ans après, le pacte est rompu par Joy V. qui décide de porter plainte pour traite des êtres humains auprès de l'OCRTEH ${ }^{38}$, l'organisme chargé de lutte contre la traite en France.

Comme de très nombreuses femmes nigérianes, Joy V. et Fatima $M$. ont été contraintes à la prostitution pour rembourser une dette allant de 30000 à 50000 euros, une somme que leurs proxénètes disent avoir dû avancer pour les frais de leur voyage. Après plus de deux ans de prostitution, elles entrent en contact avec l'association de santé communautaire par l'intermédiaire d'une amie, laquelle les encourage à porter plainte. À la suite de leurs témoignages, l'OCRTEH entame une enquête pour proxénétisme aggravé qui aboutit, en novembre 2008, à l'ouverture d'une instruction judiciaire, et en janvier 2009, à

\footnotetext{
${ }^{36}$ Collectif onze, op. cit., p. 258.

37 Élodie Apard, Precious Diagboya et Vanessa Simoni ont montré le rôle déterminant des familles et plus particulièrement des mères, dans les projets de départ. Cf. Apard É., Diagboya P., Simoni V., « « La prostitution, ça ne tue pas!» Projets d'ascension sociale familiale dans le contexte de la traite sexuelle (NigeriaEurope) », Politique africaine, vol. 159, n 3, 2020, pp. 51-82.

${ }^{38}$ Office central pour la répression de la traite des êtres humains.
} 
l'interpellation de plusieurs individus pour des faits de proxénétisme. Quelques jours après leur garde à vue, l'infraction de traite est ajoutée à ce premier chef d'accusation. Les deux femmes sont entendues à cinq et trois reprises par l'OCRTEH et à deux reprises par le juge d'instruction qui ordonne une première série d'expertises psychologiques et gynécologiques. Les victimes finissent par se constituer parties civiles au procès, mais refusent de comparaître à l'audience, préférant témoigner par visioconférence. En novembre 2010, au terme d'une procédure d'une durée moyenne, le Tribunal de grande instance de Paris condamne quatre prévenus à six ans de prison fermes, assortis d'une «invitation à quitter le territoire ». La chambre correctionnelle du TGI de Paris condamne par ailleurs les quatre prévenus à restituer 76800 et 85000 euros aux victimes. Comparé à d'autres procès pour proxénétisme aggravé où des sommes de 30000 euros sont généralement fixées, ce montant est relativement élevé, sans doute parce que l'incrimination pour «traite » a été retenue. L'avocate Claire B. estime cependant ce montant insuffisant et décide de porter le dossier devant la CIVI de Paris. En 2017, au terme d'une procédure qui a duré sept ans, Joy V. et Fatima M. obtiennent chacune 150000 euros de dédommagement.

\section{Expertiser}

Suivi social, identification, dépôt de plainte, procès et condamnation : toutes les épreuves d'un parcours de victime de traite sont ici réunies. Le dossier est par ailleurs porté dans un moment où les disputes opposant l'avocate et le Fonds de garantie franchissent une nouvelle étape et se standardisent. L'avocate sait que les montants demandés seront rabaissés, et anticipe le travail de minoration de la partie adverse, comme celle-ci se prépare à faire face à des chiffres gonflés. Les disputes se routinisent, et entraînent dans le même temps les victimes « dans un tunnel dont elles voient difficilement le bout». À l'instar des victimes de distilbène étudiées par Emmanuelle Fillion et Didier Torny, l'expérience des victimes se voit marquée par une profonde « discontinuité » : « les dossiers n'avancent pas, les audiences sont repoussées, les expertises retardées $\|^{39}$.

S'agissant d'un litige qui se passe d'audiences et qui repose essentiellement sur les examens médicaux, le nombre d'expertises ordonnées frappe par sa quantité : deux au niveau de l'instruction et près de huit dans le cadre des CIVI. «L'injonction politique à se raconter » est ici exacerbée. Cependant, contrairement à l'enquête menée par Didier Fassin auprès du Fonds d'urgence sociale, à aucun moment les victimes n'expriment une «nécessité » d'obtenir une aide financière. Cette demande est toujours formulée par l'avocate ou l'association qui les accompagne. Les victimes, elles, restent silencieuses dans ce processus. Une autre différence notable avec l'enquête de Didier Fassin tient à l'absence de toute marque de compassion dans les dossiers. Le langage utilisé par les experts est au contraire celui de la mise à distance des émotions, d'une objectivation froide et détachée des dommages constatés en référence à la nomenclature "Dintilhac ». Cela n'empêche pas l'une des expertes gynécologues de nous confier, avec des mots pleins d'empathie: «J'ai rarement vu les femmes aussi anéanties ${ }^{40} »$.

Les examens gynécologiques sont courts, d'une trentaine de minutes, ceux menés par les psychologues peuvent être plus longs, jusqu'à une heure. Ils se déroulent la plupart du temps en anglais, en l'absence d'interprète, rendant l'exercice de "subjectivation » des demandeurs difficile. L'entretien s'avère déstabilisant, soit parce qu'il est réalisé par un homme, soit parce que les victimes expriment la crainte des représailles si elles «parlent

\footnotetext{
${ }^{39}$ Fillion E., Torny D., op. cit., p. 596.

${ }^{40}$ Entretien réalisé le 2 décembre 2014.
} 
trop ». La confusion entre les examens réalisés dans le cadre de l'instruction judiciaire et ceux ordonnés par les CIVI est ici palpable.

Une autre singularité de la procédure réside dans les effets de la temporalité sur les décisions expertales. Dans le présent dossier, dix ans s'étaient écoulées entre le dépôt de plainte et l'indemnisation effective des victimes. Dans ce laps de temps, le marquage social de l'argent subit une profonde mutation. Alors que le déficit fonctionnel permanent fut fixé à $10 \%$ en 2009 , il franchit la barre des $100 \%$ cinq ans plus tard au titre du «préjudice exceptionnel d'avilissement » créé par Claire B. Cette mise en barème prend en compte la définition de la prostitution comme violence ultime à l'encontre des femmes. L'argent alloué au titre $\mathrm{du}$ "préjudice exceptionnel d'avilissement» sert non seulement à réparer les dommages causés par la prostitution (troubles psychologiques, problèmes gynécologiques), mais la prostitution per se, comme phénomène attentatoire à la dignité des femmes. Le statut de victime se voit ainsi validé par le marquage.

\section{Contester}

Le Fonds de garantie procède au contre-marquage de l'argent en contestant systématiquement les avis rendus par les experts. Cette position du Fonds doit être comprise à la lumière de sa mission : celle de protéger l'argent public et de veiller à sa juste distribution en définissant les usages acceptables ou inacceptables de l'argent provenant des contribuables. Le Fonds est en effet financé par des prélèvements, obligatoires, sur les contrats d'assurance (en 2017, le montant de la taxe a été fixé à 5,90 euros par contrat). La provenance de cet argent lui fait donc revêtir des «valeurs indépendantes de sa quantité ${ }^{41}$ ». Comme le souligne Viviana Zelizer à propos de l'argent primitif, "savoir de combien d'argent on dispose importe moins que de déterminer de quel argent il s'agit ${ }^{42} »$. Il y aurait en effet un argent de "bonne » et de "mauvaise » qualité, un argent "sacré » et un argent " profane ». Celui de qualité inférieure est considéré comme "inutile ou dévalorisé, même quand il circule en grande quantité ${ }^{43}$. À l'inverse, l'argent des contribuables, considéré comme " supérieur », "vertueux » voire " sacré », appelle un usage parcimonieux, au point où le Fonds de garantie est considéré, selon les dires d'un de ses employés, " comme un modérateur un peu pingre qui ne veut pas lâcher son argent $\gg{ }^{44}$.

Dans les dossiers de traite, les définitions autour de l'usage acceptable ou inacceptable de l'argent public se jouent principalement en référence aux notions du travail salarié et de la sexualité épanouie. Par rapport au travail, la nomenclature «Dintilhac » prévoit de réparer « la perte de chance » - le travail qui aurait pu être accompli mais n'a pu l'être en raison de la contrainte exercée sur la personne - et « la perte de gains professionnels actuels et futurs » la nature des séquelles psychologiques et physiques est considérée comme obstacle à toute activité professionnelle. Pour calculer ces postes de préjudice, Claire B. se réfère généralement au salaire minimum de croissance, soit environ 1000 euros par mois, divisé de moitié «pour prendre en compte une perte de chance». Dans le dossier de Joy V. et de Fatima M., ce mode de calcul correspond au chiffrage suivant : 500 euros x 24 mois (période de prostitution) $=12000$ euros. Le même principe est appliqué à la période relative à la recherche d'un emploi : 500 euros x 37 mois $=18500$ euros. Après avoir indemnisé le passé, le dispositif entreprend d'indemniser un avenir compromis.

\footnotetext{
${ }^{41}$ Zelizer V., op. cit., p. 58.

${ }^{4}$ Ibid.

${ }^{43}$ Ibid., p. 59.

${ }^{44}$ Entretien réalisé le 9 décembre 2014.
} 
Le Fonds de garantie conteste les arguments relatifs au travail, singulièrement dans les affaires mettant en jeu des femmes qui sont arrivées, migrantes, en France. Les arguments mobilisés sont avant tout d'ordre moral et laissent supposer qu'une étrangère sans-papiers ne maîtrisant pas le français n'aurait jamais pu trouver du travail en France. Le processus de victimisation entretient, au lieu d'effacer, le stigmate de prostituée sans-papiers. Le motif de refus dans une des affaires est formulé comme suit: "Dans cette affaire, il n'est produit aucun élément permettant d'affirmer que dès son arrivée en France et au regard des circonstances économiques actuelles, Mlle O. aurait pu trouver une activité professionnelle. Pour être indemnisée la perte de chance doit être a minima certaine et réelle. Dès lors que le préjudice invoqué est purement hypothétique, il ne peut faire l'objet d'une indemnisation. [...] Arrivée en France, Mlle O. ne maitrisait pas notre langue et je vois mal comment dans ces conditions elle aurait pu exercer une profession lui procurant même un revenu de 500 euros par mois. Il ne faut pas perdre de vue que Mlle O. arrivant du Nigéria aurait dû obtenir un titre de séjour en France et une autorisation de travail, ce qui ne paraît aucunement certain $^{45}$. »

La décision se base sur un présupposé, un jugement de valeur porté sur les femmes migrantes et non sur des données objectives. Nombre de sans-papiers ne maîtrisant pas le français exercent en effet des emplois à faible rémunération pour survivre. L'énonciation de ce type de jugements complique les échanges, déjà tendus, entre le Fonds et l'avocate des victimes. L'accusation de racisme, à peine prononcée, est un implicite de leurs relations.

Outre le travail, les pratiques de marquage de l'argent ont également trait à une certaine vision de la féminité, de la condition de la femme et de la sexualité épanouie. La nomenclature «Dintilhac» appréhende dans le même mouvement la possibilité de se reproduire, d'éprouver du plaisir et de valoriser son corps. Chez certaines femmes, les effets négatifs du passé prostitutionnel continuent à affecter défavorablement le présent. Fatima M., vierge au moment de sa première passe, n'imagine pas encore " "dormir" avec un homme "; une autre a entrepris une réduction mammaire car elle "ne supportait plus le regard des hommes sur sa poitrine qu'elle jugeait trop volumineuse ». Si le Fonds reconnaît les effets négatifs du passé prostitutionnel sur les pratiques sexuelles des femmes, il appose systématiquement son refus d'indemnisation lorsque celles-ci retrouvent une sexualité régulière ou qu'elles parviennent à fonder une famille. La "sexualité épanouie » ou une « libido saine » n'étant pas définies par le droit, une certaine latitude d'interprétation est laissée aux acteurs investis dans la juste distribution d'argent public. Le marquage de l'argent résulte ainsi de cet entrelacs d'instruments de mesure standardisés et de catégories morales de jugement.

$* * *$

L'objectif du présent article a été de regarder comment un phénomène jugé dégradant pour les femmes façonne les différents usages de l'argent public. Loin d'être neutre ou anonyme, l'argent mis ici en circulation est contrôlé, rationné, voire moralisé. Ici, comme dans les cas étudiés par Viviana Zelizer, l'argent est constamment pris dans un faisceau de relations, de contraintes et de savoirs expertaux, mais aussi dans les définitions concurrentes du propre et du sale, des vies précaires et des vies bonnes, des dignités brisées et celles qu'il convient de redresser. Les CIVI parviennent finalement à transformer la signification sociale de l'argent issu du proxénétisme et de la traite, en le redistribuant aux victimes sur une échelle de valeurs relatives au travail et à la sexualité. Le processus de victimisation ne parvient

\footnotetext{
${ }^{45}$ Il s'agit du dossier d'une femme originaire du Nigéria qui s'est prostituée en France entre 2005 et 2007.
} 
cependant pas à effacer complètement le stigmate de prostituée et de sans-papiers qui continue à peser négativement sur la « mise en barème » des souffrances endurées.

Reste à savoir quel usage font les principales intéressées de l'argent ainsi distribué. Il n'est en effet pas rare que les victimes de traite, considérées comme " dépensières », soient mises sous curatelle et accompagnées par une personne qui les aide à ne pas «tout dilapider ${ }^{46}$ ». Sollicitées par leurs familles restées au pays et soupçonnées ignorer la «valeur de l'argent », leur usage des sommes allouées par le Fonds de garantie fait parfois l'objet d'un apprentissage, comme si le fait de donner de l'argent liquide aux personnes jugées pauvres était considéré comme potentiellement dangereux. Le parcours des victimes de traite se poursuit donc au-delà de cette dernière épreuve. Ce temps long de la victimisation ouvre la question de savoir s'il est possible de rompre avec le statut de victime et à quelle condition.

\footnotetext{
${ }^{46}$ Entretien avec une avocate des victimes, 5 mars 2015.
} 